\title{
Oblique-incidence reflectometry: one relative profile measurement of diffuse reflectance yields two optical parameters
}

Lihong V. Wang, Shao-Pow Lin, Steven L. Jacques, Frank K. Tittel, Jennifer Harder, et al.

Lihong V. Wang, Shao-Pow Lin, Steven L. Jacques, Frank K. Tittel, Jennifer Harder, John Jancarik, Beth Michelle Mammini, Ward Small, Luiz Barroca Da Silva, "Oblique-incidence reflectometry: one relative profile measurement of diffuse reflectance yields two optical parameters," Proc. SPIE 2627, Optical Biopsies, (27 December 1995); doi: 10.1117/12.228887

SPIE. Event: BiOS Europe '95, 1995, Barcelona, Spain 


\title{
Oblique-Incidence Reflectometry: One Relative Profile Measurement of Diffuse Reflectance Yields Two Optical Parameters
}

\author{
Lihong Wang, Ph. D. (1)*, Shao-Pow Lin, B. S. $(1,2)$ \\ Steven L. Jacques, Ph.D. (1), Frank K. Tittel, Ph. D. (2) \\ Jennifer Harder, B. S. (3), John Jancarik, B. S. (3) \\ Beth Mammini, B. S. (3), Ward Small, B. S. (3), Luiz Da Silva, Ph. D. (3) \\ (1) Laser Biology Research Laboratory \\ University of Texas M. D. Anderson Cancer Center \\ 1515 Holcombe Boulevard, Houston, Texas 77030, USA \\ (2) Department of Electrical and Computer Engineering \\ Rice University, Houston, Texas, USA \\ (3) Laser Program, Lawrence Livermore National Laboratory \\ Livermore, California, USA
}

\begin{abstract}
A new, simple and quick approach, oblique-incidence reflectometry, was used to measure the absorption and reduced scattering coefficients of a semi-infinite turbid medium. An obliquely incident light beam causes the center of the far diffuse reflectance to shift from the point of incidence, where the far diffuse reflectance refers to the diffuse reflectance that is several transport mean free paths away from the incident point. The amount of shift yields the diffusion constant by a simple formula, and the slope of the diffuse reflectance yields the attenuation coefficient. Only the relative profile of the diffuse reflectance is needed to deduce both optical parameters, which makes this method attractive in clinical settings because it does not require a stringent calibration for absolute quantity measurements. This method was tested theoretically by Monte Carlo simulations and experimentally by a reflectometer. Because this method can be used to measure optical properties of biological tissues quickly and requires on inexpensive equipment, it has potential clinical application to the diagnosis of disease or monitoring of treatments.
\end{abstract}

\section{Key Words}

Oblique incidence, reflectometry, optical properties, turbid media, biological tissues.

* To whom correspondence should be addressed. Assistant Professor.

Emails: lihong@laser.mda.uth.tmc.edu

Phone: (713) 745-1742 Fax: (713) 792-3995 


\section{Introduction}

In both diagnostic and therapeutic applications of lasers or other light sources in medicine, it is important to evaluate the optical properties of biological tissues, which are turbid media. The optical properties can be used to diagnose diseases, to measure tissue metabolic status, or to determine the dosage in therapeutic applications of lasers. Non-invasive, in vivo, real-time measurements of tissue optical properties, the absorption coefficient $\mu_{\mathrm{a}}$ and reduced scattering coefficient $\mu_{\mathrm{s}}^{\prime}$, remain a challenge.

For simplicity, we will define the diffuse reflectance that is several transport mean free $(\mathrm{mfp})$ away from the incident point of the laser beam as far diffuse reflectance. Conversely, we will define the diffuse reflectance that is within several mfp' away from the incident point of the laser beam as near diffuse reflectance. A typical diffuse reflectance profile of a laser beam normally incident upon a semi-infinite turbid medium, $\mathrm{R}(\mathrm{x})$, is shown in Fig. 1. Monte Carlo ${ }^{1}$ simulated diffuse reflectance is considered accurate, whereas the diffusion theory predicted diffuse reflectance is only accurate for the far diffuse reflectance.

The far diffuse reflectance becomes linear on a semi-logarithmic plot, and the slope of the diffuse reflectance is primarily determined by the effective attenuation coefficient, $\mu_{\text {eff }}$. If one measures the relative profile of the diffuse reflectance $R(x)$, the effective attenuation coefficient can be computed approximately using a relatively simple algorithm based on the

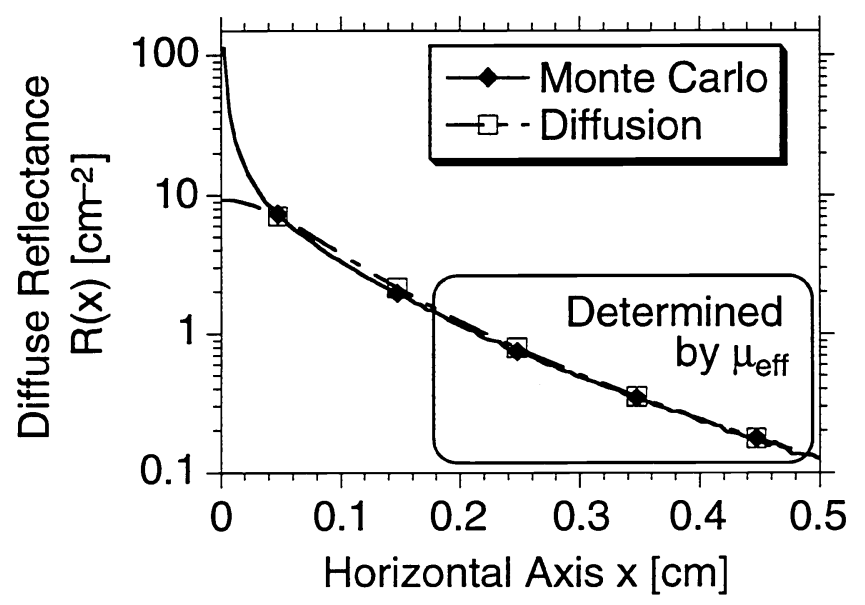

Fig. 1. Diffuse reflectance vs. horizontal axis $x$. The laser beam is normally incident upon a semi-infinite turbid medium. The horizontal axis $\mathrm{x}$ is the distance between the observation point and the incident point of the laser beam on the surface. The computations were based on Monte Carlo simulation and diffusion theory. The optical properties of the turbid medium are index of refraction $n=1.0$, absorption coefficient $\mu_{\mathrm{a}}=0.1 \mathrm{~cm}^{-1}$, scattering coefficient $\mu_{\mathrm{s}}=100 \mathrm{~cm}^{-1}$, and anisotropy $\mathrm{g}=0.9$. 
slope of the reflectance. ${ }^{2}$ If we wish to measure both absorption and reduced scattering coefficients, two independent parameters have to be determined. The near diffuse reflectance of the relative profile has to be used to determine another independent parameter, which creates a dilemma. No simple algorithm is available to deduce the second parameter from the near diffuse reflectance. Iterative algorithms based on Monte Carlo simulations will be time-consuming because Monte Carlo simulations are slow. Neural network may be used to solve the inverse problem after the network is trained on a set of Monte Carlo simulation results of various optical properties. ${ }^{2,3}$ Once the neural network is trained, the inverse problem can be solved in real-time. However, the neural network approach involves a long training process.

Algorithms based on diffusion theory cannot use the near diffuse reflectance because of the inaccuracy of diffusion theory in the near region. If the absolute far diffuse reflectance is measured, the diffusion theory based algorithms can be used to deduce the second parameter using non-linear least squares fits of the far diffuse reflectance. However, in clinical settings it is much easier to measure the relative profile of diffuse reflectance than to measure the absolute profile.

Oblique-incidence reflectometry (Fig. 2) deduces diffusion constant very quickly and is independent of the laser beam size if (1) the laser beam has a predefined mirror symmetry and (2) the size of the beam is smaller than the distance between the measurement points and the center of the laser beam. ${ }^{4,5}$ For example, a circular flat beam or a circular Gaussian beam

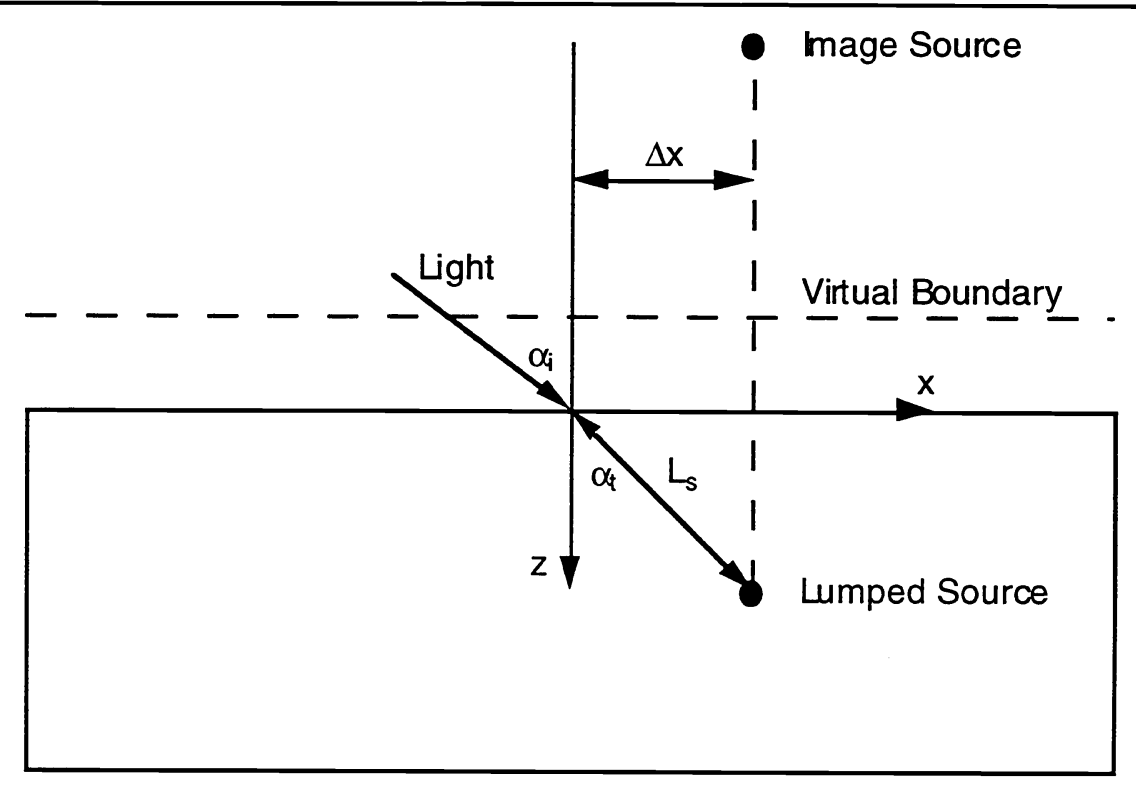

Fig. 2. Coordinate system and the lumped interaction approximation of the laser beam. The origin of the coordinate system is at the incident point of the light beam. In the figure, $\alpha_{i}$ is the incident angle of the laser beam, $\alpha_{t}$ is the refracted angle, $\Delta x$ is the horizontal shift of the lumped interaction site from the incident point. The $y$-axis point outward from the paper. $L_{s}=1 /\left(0.35 \mu_{a}+\mu_{s}^{\prime}\right)$. 
has an elliptical spot on the medium surface when the beam is incident at an oblique angle. The mirror symmetry of this elliptical spot about its short axis allows the application of this method to measure $\mu_{\mathrm{s}}^{\prime}$. The method was verified theoretically and experimentally.

The diffuse reflectance with the oblique incidence is shifted toward the $+x$ direction. The amount of shift is computed by

$$
\Delta \mathrm{x}=\frac{\sin \left(\alpha_{\mathrm{i}}\right)}{\mathrm{n}\left(0.35 \mu_{\mathrm{a}}+\mu_{\mathrm{s}}{ }^{\prime}\right)}=\frac{\sin \left(\alpha_{\mathrm{s}}\right)}{0.35 \mu_{\mathrm{a}}+\mu_{\mathrm{s}}{ }^{\prime}},
$$

as derived in our previous publication. ${ }^{4}$

Here we will present more experimental results using an optical fiber probe to deduce two optical parameters: (1) the shift of far diffuse reflectance, $\Delta x$; and (2) the effective attenuation coefficient, $\mu_{\text {eff }}$. Based on these two parameters, we can compute for the absorption and reduced scattering coefficients, $\mu_{\mathrm{a}}$ and $\mu_{\mathrm{s}}{ }^{\prime}$.

\section{Methods and Materials}

\section{Experimental}

An oblique-incidence optical-fiber probe was built. The tips of eleven optical fibers were sanded at 45 degrees. One optical fiber was used as the source to deliver laser light (632.8 $\mathrm{nm}$ wavelength, He-Ne laser) to the turbid media, and the other ten were used as the detection fibers to collect light from the turbid media. The light from the detection fibers was measured using an optical power meter. The eleven fibers were bundled together in plastic to form a hand-held probe (Fig. 3). A thin layer of diffuser was added to each of the ten detection fibers. The distances between the detection fibers and the source fiber are listed in Table 1. Each detection fiber was then calibrated for its relative transmission of light.

Table 1. Probe configuration.

\begin{tabular}{|r|c|}
\hline Detection Fiber & Distance from Source $(\mathrm{cm})$ \\
\hline Leftmost, 1 & -0.800 \\
\hline 2 & 0.224 \\
\hline 3 & 0.476 \\
\hline 4 & 0.735 \\
\hline 5 & 0.991 \\
\hline 6 & 1.245 \\
\hline 7 & 1.503 \\
\hline 8 & 1.747 \\
\hline 9 & 1.974 \\
\hline Rightmost, 10 & 2.265 \\
\hline
\end{tabular}




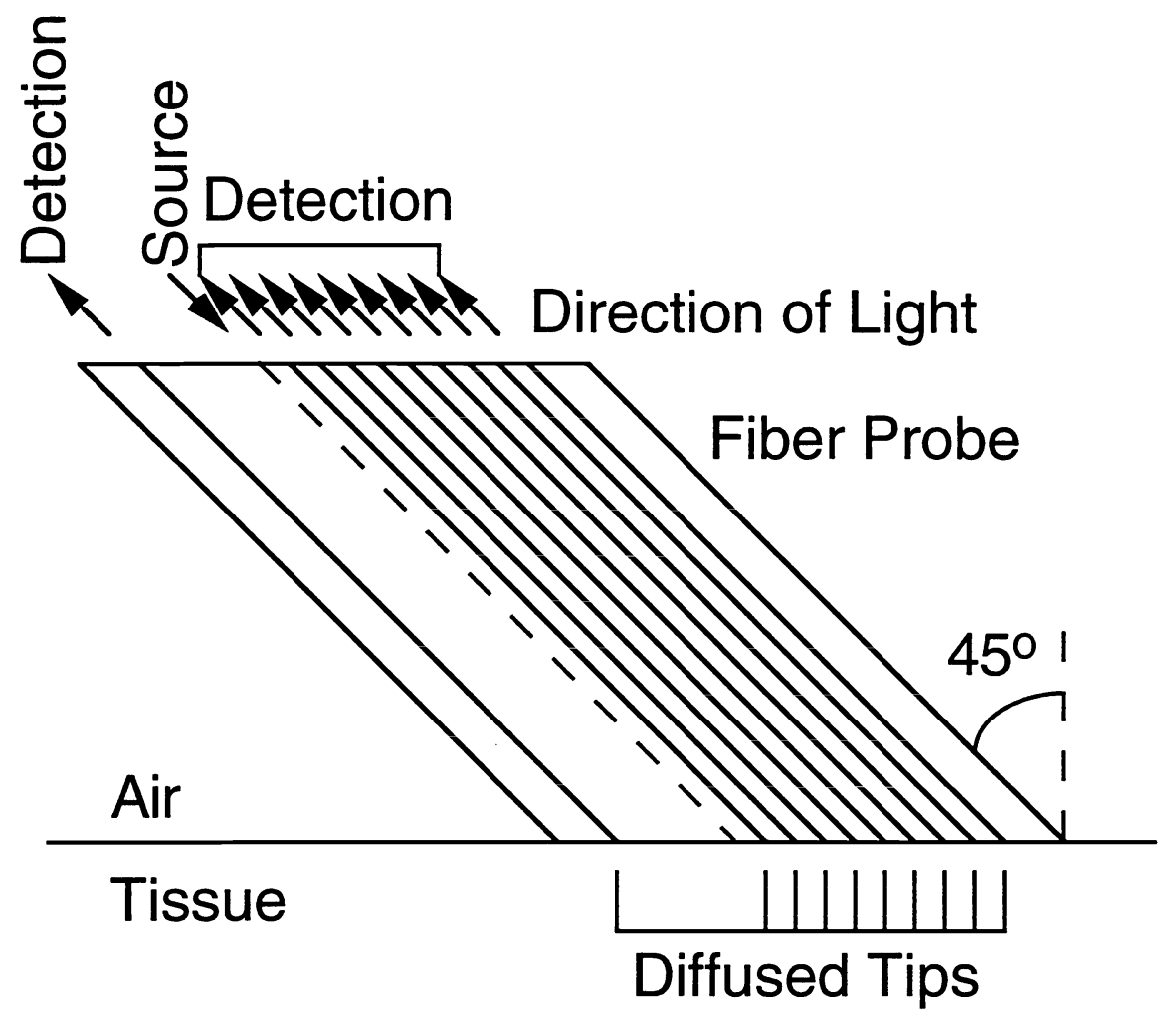

Fig. 3. An oblique-incidence optical fiber probe. One source fiber and ten detection fibers were bundled and encased to form a hand-held piece. The core diameter of the fibers was $600 \mu \mathrm{m}$. The incident angle of the probe was $45^{\circ}$. The index of refraction of the fiber core was 1.46. The index of refraction of tissue phantom was 1.33. The angle of refraction in a liquid tissue phantom was computed to be $50.9^{\circ}$.

Turbid media simulating the optical properties of biological tissues (tissue phantoms) were made of water, Trypan blue dye, and polystyrene spheres. The Trypan blue dye was dominantly absorbing and had negligible scattering. The polystyrene spheres $(899.5 \mathrm{~nm}$ diameter) were dominantly scattering and had little absorption. The absorption coefficient of the Trypan blue dye stock solution was measured using a spectrophotometer. The scattering anisotropy of the polystyrene spheres was computed using Mie theory, ${ }^{6}$ and the scattering coefficient was obtained using a collimated transmission measurement. ${ }^{7}$ Different concentrations of the dye and the spheres can be mixed into water to make a solution of desired optical properties. The tissue phantom presented here has the following optical properties: $\mu_{\mathrm{a}}=$ $0.40 \mathrm{~cm}^{-1}, \mu_{\mathrm{s}}{ }^{\prime}=4.0 \mathrm{~cm}^{-1}$, and $\mathrm{g}=0.914$ at $632.8 \mathrm{~nm}$ wavelength.

\section{Theoretical}

Diffusion theory was used to fit the measured diffuse reflectance for the effective attenuation coefficient, $\mu_{\text {eff }}$, whereas the shift of diffuse reflectance, $\Delta x$, provided another 
equation (Eq. 1). The two equations were used to solve for the absorption and reduced scattering coefficients, $\mu_{\mathrm{a}}$ and $\mu_{\mathrm{s}}$.

The diffusion theory is based on the extrapolated boundary condition and lumped interaction approximation of the incident light. ${ }^{8,9}$ Traditionally, the lumped point source is placed a distance $=1 /\left(\mu_{\mathrm{a}}+\mu_{\mathrm{s}}{ }^{\prime}\right)$ into turbid media from the entry point. However, Eq. 1 indicates that the source should be placed a distance $=1 /\left(0.35 \mu_{\mathrm{a}}+\mu_{\mathrm{s}}{ }^{\prime}\right)$ from the entry point, instead. Therefore, the depth of the lumped source is

$$
\Delta \mathrm{z}=\frac{\cos \left(\alpha_{\mathrm{t}}\right)}{0.35 \mu_{\mathrm{a}}+\mu_{\mathrm{s}}{ }^{\prime}}=\Delta \mathrm{x} \operatorname{ctan}\left(\alpha_{\mathrm{t}}\right)
$$

Similarly, we have found that the diffusion constant should be

$$
\mathrm{D}=\frac{1}{3\left(0.35 \mu_{\mathrm{a}}+\mu_{\mathrm{s}}{ }^{\prime}\right)}=\frac{\Delta \mathrm{x}}{3 \sin \left(\alpha_{\mathrm{t}}\right)} \approx \frac{1}{\mu_{\mathrm{a}}+3 \mu_{\mathrm{s}}{ }^{\prime}}
$$

Eq. 3 improves the accuracy of diffusion theory when compared with Monte Carlo simulations. ${ }^{10}$

First, the shift, $\Delta \mathrm{x}$, was computed from the measured diffuse reflectance (Fig. 4). A Cprogram searched an interaction point on the right-hand side of the diffuse reflectance curve such that the diffuse reflectance at this point was equal to that of the leftmost data point. The mid-point between the intersection point and the leftmost data point yielded $\Delta \mathrm{x}$.

Then, the following diffusion theory was used to fit for $\mu_{\text {eff }}$ based on the LevenbergMarquardt method, which is a non-linear least-squares fitting method. ${ }^{11}$ The average internal reflection is ${ }^{12}$

$$
\mathrm{r}_{\mathrm{i}}=-1.440 \mathrm{n}_{\mathrm{rel}}^{-2}+0.710 \mathrm{n}_{\text {rel }}^{-1}+0.668+0.0636 \mathrm{n}_{\text {rel }} \text {, }
$$

where the relative refractive index, $\mathrm{n}_{\text {rel }}$, is the ratio between the refractive indices of the turbid medium and ambient medium.

$$
A=\left(1+r_{j}\right) /\left(1-r_{i}\right)
$$

The distance between the virtual boundary and the surface of the turbid medium is

$$
z_{b}=2 A D
$$

The fluence on the virtual boundary is approximately zero. The distance between the observation point $(\mathrm{x}, 0,0)$ and the lumped source $(\Delta \mathrm{x}, 0, \Delta \mathrm{z})$ is

$$
\rho_{1}=\sqrt{(x-\Delta x)^{2}+\Delta z^{2}} \text {. }
$$

The distance between the observation point $(\mathrm{x}, 0,0)$ and the image source $\left(\Delta \mathrm{x}, 0,-\Delta \mathrm{z}-2 \mathrm{z}_{\mathrm{b}}\right)$ is 


$$
\rho_{2}=\sqrt{(x-\Delta x)^{2}+\left(\Delta z+2 z_{b}\right)^{2}} \text {. }
$$

The diffuse reflectance is

$$
R(x)=\frac{\Delta z\left(1+\mu_{\text {eff }} \rho_{1}\right) \exp \left(-\mu_{\text {eff }} \rho_{1}\right)}{4 \pi \rho_{1}{ }^{3}}+\frac{\left(\Delta z+2 z_{b}\right)\left(1+\mu_{\text {eff }} \rho_{2}\right) \exp \left(-\mu_{\text {eff }} \rho_{2}\right)}{4 \pi \rho_{2}^{5}}
$$

Because the measured data represent the relative profile of diffuse reflectance, $k \mathrm{R}(\mathrm{x})$ was used to fit for the effective attenuation coefficient, where $\mathrm{k}$ is a scaling factor. Because diffusion theory does not hold for $|\mathrm{x}|<1 \mathrm{mfp}$, any data experimental points falling into this region should be excluded from the fitting.

When $\mu_{\text {eff }}$ was obtained from the curve fitting, Eq. 1 and the following equation were used to compute for $\mu_{\mathrm{a}}$ and $\mu_{\mathrm{s}}$ '.

$$
\mu_{\mathrm{eff}}=\sqrt{\mu_{\mathrm{a}} / \mathrm{D}}=\sqrt{\frac{3 \sin \left(\alpha_{\mathrm{t}}\right) \mu_{\mathrm{a}}}{\Delta \mathrm{x}}} .
$$

or

$$
\mu_{\mathrm{a}}=\frac{\mu_{\text {eff }}^{2} \Delta \mathrm{x}}{3 \sin \left(\alpha_{\mathrm{t}}\right)}
$$

and

$$
\mu_{\mathrm{s}}^{\prime}=\frac{\sin \left(\alpha_{\mathrm{t}}\right)}{\Delta \mathrm{x}}-0.35 \mu_{\mathrm{a}}
$$

\section{Results}

A measured diffuse reflectance curve was fitted using the above theory (Fig. 4). The fitted results were compared with the expected values based on the optical properties of the tissue phantom (Table 2). The relative errors were below 10\%. The non-linear least-squares fit

Table 2. Comparison between measured and expected results.

\begin{tabular}{|l|l|l|l|}
\hline Parameter & Expected & Fitted & Relative Error \\
\hline$\Delta \mathrm{x}(\mathrm{cm})$ & 0.187 & 0.204 & $8.6 \%$ \\
\hline$\mu_{\mathrm{eff}}\left(\mathrm{cm}^{-1}\right)$ & 2.229 & 2.118 & $-5.0 \%$ \\
\hline$\mu_{\mathrm{a}}\left(\mathrm{cm}^{-1}\right)$ & 0.40 & 0.392 & $-2.0 \%$ \\
\hline$\mu_{\mathrm{s}}{ }^{\prime}\left(\mathrm{cm}^{-1}\right)$ & 4.0 & 3.675 & $-8.1 \%$ \\
\hline
\end{tabular}




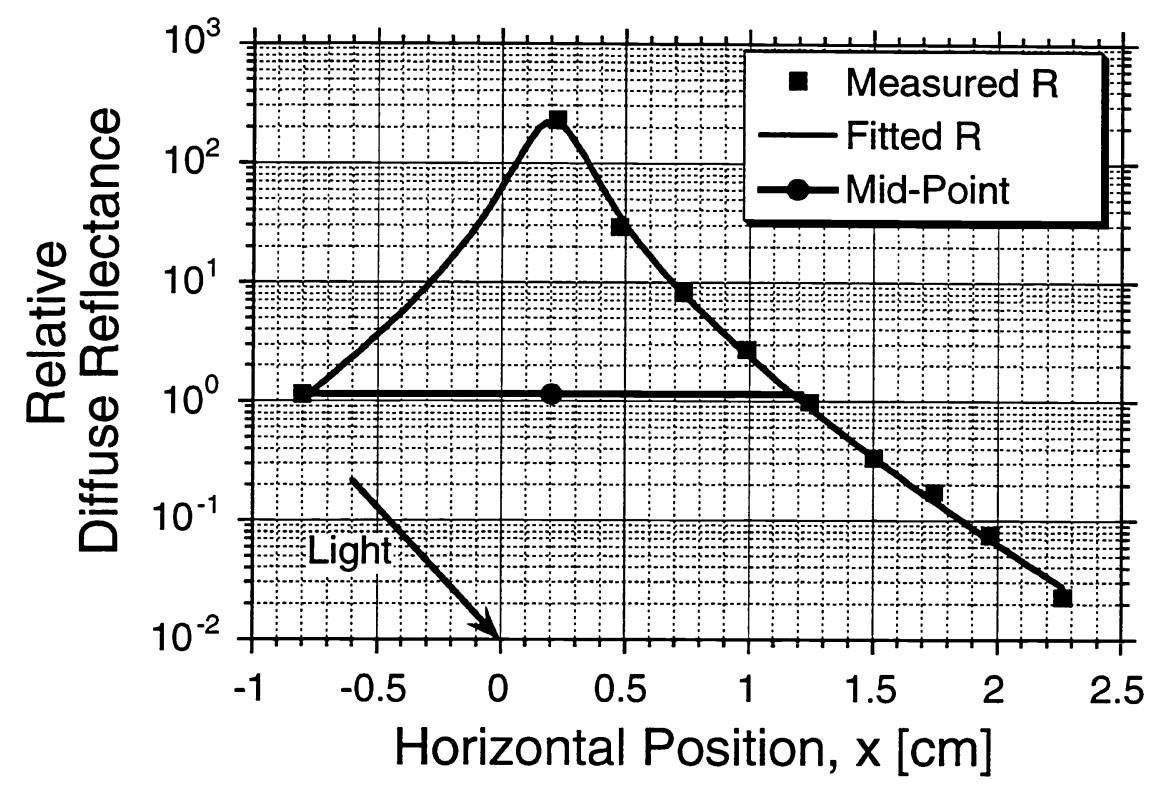

Fig. 4. The measured and fitted diffuse reflectance and the fitted shift, $\Delta x$. The experimental data were measured using the optical fiber probe on a tissue phantom. The horizontal positions of the measured data correspond to the positions of the detection fibers (Table 1). The fitted shift was the mid-point of the horizontal line connecting the leftmost measured data point and the intersection between the line and the right side of the measured diffuse reflectance.

took $<0.1 \mathrm{sec}$ on a Sun SPARCstation 2 computer. A refractive-index-matched boundary condition was used for the fitting.

\section{Discussion and Conclusions}

The boundary condition that was used for the curve fitting was refractive-index matched. The refractive indices of both the ambient medium and the turbid medium were set to 1.33 , which is the refractive index of water. The surface of the turbid medium was partially exposed to air and partially exposed to the probe. It is inappropriate to use the refractive index of either air (1.0) or probe material (1.45, plastic) for the ambient medium. Before a better solution becomes available, we used 1.33 as the refractive index of the ambient medium. The fitted results seemed to be in good agreement with the expected results maybe partly because 1.33 was between 1.0 and 1.45 .

The optical probe based on oblique-incidence reflectometry allows us to quickly measure both absorption and reduced scattering coefficients of turbid media. The shift of the diffuse reflectance with respect to the light entry point is sensitive to the diffusion constant. The slope of the diffuse reflectance is sensitive to the effective attenuation coefficient. Therefore, 
these two parameters $\left(\mu_{\mathrm{a}}\right.$ and $\left.\mu_{\mathrm{s}}{ }^{\prime}\right)$ can measured using the relative profile of the diffuse reflectance with oblique-incident light.

We are extending the current probe to a multi-wavelength probe, which is able to measure the optical properties of turbid media at multiple wavelengths simultaneously. A filtered arc-lamp will be the light source. The white light collected by the detection fibers will be expanded spectrally through a spectrograph and then projected onto a $\mathrm{CCD}$ matrix. One dimension of the CCD matrix represents the spatial information of the diffuse reflectance, while the other the spectral information. The above procedure will be used to compute the optical properties at each wavelength rapidly.

\section{Acknowledgment}

The project was sponsored in part by The Whitaker Foundation, Air Force Office of Scientific Research grant F49620-93-1-0298DEF, Department of Energy grant DE-FG0591ER61226, and National Institutes of Health grant R29-HL45045. 


\section{GLOSSARY}

\begin{tabular}{|c|c|}
\hline $\begin{array}{l}\text { Absorption coefficient } \\
{\left[\mu_{\mathrm{a}}, \mathrm{cm}^{-1}\right]}\end{array}$ & $\begin{array}{l}\text { The probability of photon absorption per unit infinitesimal } \\
\text { pathlength. }\end{array}$ \\
\hline Anisotropy $[\mathrm{g}]$ & $\begin{array}{l}\text { The average of the cosine value of the deflection angle by single } \\
\text { scattering. }\end{array}$ \\
\hline $\begin{array}{l}\text { Diffusion constant } \\
{[\mathrm{D}, \mathrm{cm}]}\end{array}$ & $\begin{array}{l}\text { Linking the gradient of light fluence, } \nabla \phi \text {, and light current, } \mathrm{F} \text {, } \\
\text { (Fick's law), i.e., } \mathrm{F}=-\mathrm{D} \nabla_{\phi} \text {. }\end{array}$ \\
\hline $\begin{array}{l}\text { Effective attenuation } \\
\text { coefficient }\left[\mu_{\mathrm{eff}}, \mathrm{cm}^{-1}\right]\end{array}$ & $\begin{array}{l}\text { The decay constant of light fluence far away from light source. } \\
\mu_{\text {eff }}=\sqrt{3 \mu_{\mathrm{a}} / \mathrm{D}} \text {. }\end{array}$ \\
\hline $\begin{array}{l}\text { Interaction coefficient } \\
{\left[\mu_{\mathrm{t}}, \mathrm{cm}^{-1}\right]}\end{array}$ & $\begin{array}{l}\text { The probability of photon interaction per unit infinitesimal } \\
\text { pathlength, where the interaction includes both absorption and } \\
\text { scattering. } \mu_{\mathrm{t}}=\mu_{\mathrm{a}}+\mu_{\mathrm{s}} \text {. Sometimes, it is also called total } \\
\text { interaction coefficient or total attenuation coefficient. }\end{array}$ \\
\hline Mean free path $[\mathrm{mfp}]$ & The mean pathlength between interactions, which is $1 / \mu_{\mathrm{t}}$. \\
\hline $\begin{array}{l}\text { Penetration depth }[\delta \\
\mathrm{cm}]\end{array}$ & $\begin{array}{l}\delta=1 / \mu_{\text {eff. }} \text { It represents decay constant of the light fluence far from } \\
\text { the source. }\end{array}$ \\
\hline $\begin{array}{l}\text { Reduced scattering } \\
\text { coefficient }\left[\mu_{\mathrm{s}}^{\prime}, \mathrm{cm}^{-1}\right]\end{array}$ & $\begin{array}{l}\mu_{\mathrm{s}}^{\prime}=\mu_{\mathrm{s}}(1-\mathrm{g}) . \text { Sometimes, it is also called transport scattering } \\
\text { coefficient. }\end{array}$ \\
\hline $\begin{array}{l}\text { Scattering coefficient } \\
{\left[\mu_{s}, \mathrm{~cm}^{-1}\right]}\end{array}$ & $\begin{array}{l}\text { The probability of photon scattering per unit infinitesimal } \\
\text { pathlength. }\end{array}$ \\
\hline $\begin{array}{l}\text { Transport interaction } \\
\text { coefficient }\left[\mu_{\mathrm{t}}^{\prime}, \mathrm{cm}^{-1}\right]\end{array}$ & $\mu_{\mathrm{t}}^{\prime}=\mu_{\mathrm{a}}+\mu_{\mathrm{s}}^{\prime}$. \\
\hline $\begin{array}{l}\text { Transport mean free } \\
\text { path [mfp'] }\end{array}$ & $1 / \mu_{\mathrm{t}}^{\prime}$ \\
\hline
\end{tabular}




\section{References}

1. L.-H. Wang, S. L. Jacques, and L.-Q. Zheng, "MCML - Monte Carlo modeling of photon transport in multi-layered tissues," Computer Methods and Programs in Biomedicine 47, 131-146 (1995).

2. T. J. Farrell, B. C. Wilson, and M. S. Patterson, "The use of a neural network to determine tissue optical properties from spatially resolved diffuse reflectance measurements," Phys. Med. Biol. 37, 2281-2286 (1992).

3. L.-H. Wang, X.-M. Zhao, and S. L. Jacques, "Computation of the optical properties of tissues from light reflectance using a neural network," Proc. Soc. Photo-Opt. Instrum. Eng. 2134, 391-399 (1994).

4. L.-H. Wang and S. L. Jacques, "Use of a laser beam with an oblique angle of incidence to measure the reduced scattering coefficient of a turbid medium," Applied Optics 34, 2362-2366 (1995).

5. L.-H. Wang and S. L. Jacques, "Using an obliquely incident laser beam to measure optical properties of turbid media," Proc. Soc. Photo-Opt. Instrum. Eng. 2389, 522532 (1995).

6. L.-H. Wang and S. L. Jacques, MIESPHR Program: Mie Theory for Scattering Spherical Particles (University of Texas M.D. Anderson Cancer Center, Houston, Texas, USA, 1995). Downloadable using anonymous ftp from laser.mda.uth.tmc.edu (129.106.60.92).

7. L.-H. Wang and S. L. Jacques, "Error estimation of measuring total interaction coefficients of turbid media using collimated light transmission," Physics in Medicine and Biology 39, 2349-2354 (1994).

8. T. J. Farrell, M. S. Patterson, and B. C. Wilson, "A diffusion theory model of spatially resolved, steady-state diffuse reflectance for the non-invasive determination of tissue optical properties in vivo," Med. Phys. 19, 879-888 (1992).

9. L.-H. Wang and S. L. Jacques, "Analysis of diffusion theory and similarity relations," Proc. Soc. Photo-Opt. Instrum. Eng. 1888, 107-116 (1993).

10. S. L. Jacques, M. R. Ostermeyer, and L.-H. Wang, "On the diffusion constant of light transport in turbid media," in preparation (1995).

11. W. H. Press, B. P. Flannery, S. A. Teukolsky, and W. T. Vetterling, Numerical Recipes in C, 2nd ed. (Cambridge University Press, 1992).

12. R. A. J. Groenhuis, H. A. Ferwerda, and J. J. Ten Bosch, "Scattering and absorption of turbid materials determined from reflection measurements. I: theory," Applied Optics 22, 2456-2462 (1983). 